\title{
Apparatus to Measure Small-Angle Light Scattering Profiles of Polymers under Shear Flow
}

\author{
Takeji Hashimoto, Tomoaki TAKebe, and Shoji Suehiro \\ Department of Polymer Chemistry, Faculty of Engineering, \\ Kyoto University, Kyoto 606, Japan
}

(Received November 5, 1984)

\begin{abstract}
The apparatus and the principle to measure flow small-angle light scattering ("flow-SALS") are presented as one of the rheo-optical techniques. The flow-SALS technique presented here enables one to investigate small-angle light scattering profiles and rheological properties of polymer solutions, liquids, and mixtures, as well as polymer liquid crystals under various types of shearing, i.e., steady-state shear flow, oscillatory shear flow, and shear recovery after the cessation of steady-state flow. This technique may be useful to investigate the flow-induced formation, dissolution, and deformation of supermolecular structure with spatial correlation length of a few microns, and also their effect on rheological properties. In order to demonstrate the prospect of its use, some preliminary results on flow-SALS from the lyotropic polymer liquid crystal $[20 \mathrm{wt} \% \operatorname{poly}(\gamma$-methyl-D-glutamate $)$ in $m$-cresol] are presented.

KEY WORDS Small-Angle Light Scattering / Rheology / Rheo-Optics / Flow Small-Angle Light Scattering / Cone-Plate Viscometer / Steady State Shear Flow / Recovery from Shear Deformation / Lyotropic Polymer Liquid Crystal / Flow Induced Phase Transition /
\end{abstract}

Rheo-optical techniques which simultaneously give information on rheological and optical properties have been proven to be useful for obtaining deep insights into the molecular and structural origin of rheological phenomena. ${ }^{1.2}$ These techniques are especially useful for polymeric systems with supermolecular structures in solutions, liquids, and solids.

Rheo-optics has first been applied extensively to polymers in the solid state ${ }^{1,2}$ and recently to polymer liquid crystals. $^{3-15}$ Pioneering works on the rheo-optics of the liquid crystals have been initiated by Pochan et $a l^{3-5}$ who measured spectra for the transmitted light intensity of cholesteric liquid crystals, cholesteric-nematic mixtures, and cholesteric polymer blends as a function of shear rate. They interpreted the rheological data in the light of the optical data. Asada et al. ${ }^{6,14}$ later applied this method extensively to polymer liquid crystals. They developed rheo-optical techniques to measure flow birefringence and transmitted light intensity under parallel and cross-polarizers, and also simultaneously mea-

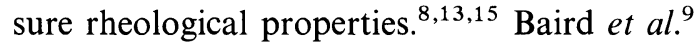
investigated flow birefringence and rheology for polymer liquid crystals. Horio, ${ }^{7}$ Kiss et al., ${ }^{11}$ and Aoki et al. ${ }^{10,12}$ conducted a different type of rheo-optical studies of polymer liquid crystals by observing their textures in external mechanical fields directly under optical microscope.

This paper presents a new rheo-optical technique by which the rheological properties of flowing polymeric systems in liquids and solutions are investigated simultaneously with depolarized and polarized components of small-angle laser light scattering. The flow small-angle light scattering technique presented here (designated hereafter as "flowSALS") may have fundamental differences 
with a conventional flow light scattering method in the sense that in the conventional flow light scattering, one usually investigates scattering from molecularly dispersed systems or individual macromolecules subjected to shear flow, while in the flow-SALS, one investigates scattering from molecular aggregates under shear flow. This technique will be widely applicable to the flow induced formation, dissolution, and deformation of molecular aggregates, and flow-induced phase transitions of liquid crystals and of multicomponent polymer mixtures as well.

Although the flow-SALS technique has never been reported or investigated previously, it is believed to give direct information on the formation and dissolution of supermolecular structure under the shear flow. This is because the elastic light scattering depends on spatial correlations of concentration fluctuations and on orientation fluctuations of optical axes of anisotropic scatterers as well as the average orientation of the optical axes. $^{2,16,19-25,28,30-33}$

In comparison, the flow-birefringence technique only gives information on the average orientation of the optical axes. Thus in principle, the flow-SALS technique provides much more information than the flow-birefringence or transmittance technique. The flow-SALS technique gives fundamental information on the nature of supermolecular structures which exist under the shear flow, i.e., the information as to whether the structures originate from concentration fluctuations or from orientation fluctuations.

Therefore, this technique is believed to be important and must be thoroughly explored in the future. In this paper we describe apparatus used for investigating the flow-SALS and give preliminary experimental results to demonstrate its possible usefulness. Further discussion on full results (and analyses of the experimental results) are beyond the scope of this paper, and will be presented in subsequent communications.

\section{FLOW-SALS APPARATUS}

The flow-SALS apparatus was constructed by modifying an existing rheometer (IR-200, Iwamoto Seisakusho Co., Ltd., Kyoto, Japan). Figure 1 shows a schematic diagram of the apparatus. The rheometer can measure rheological properties of polymers under steady-state and oscillatory shear flow, under "saw-tooth" shear flow consisting of two periods with linearly increasing and decreasing rates, and during recovery after cessation of the shear flow. The flow-SALS measurements were made possible by using a set of optically transparent cone (E) and plate $(G)$ or of parallel plates made of quartz. The data reported here were obtained with the cone and plate with radius of $40 \mathrm{~mm}$ and cone angle of 3.313 degrees.

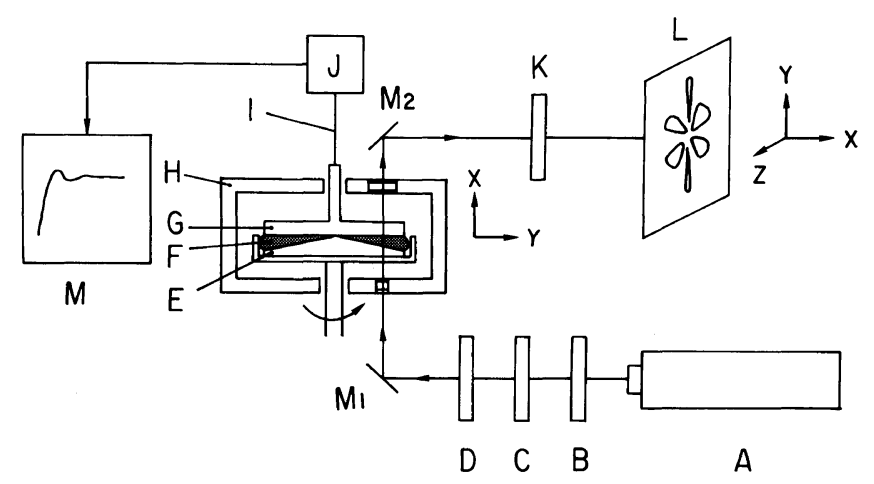

Figure 1. Schematic representation of the flow small-angle laser light scattering apparatus. 
The sample $(\mathrm{F})$, cone, and plate were covered by a temperature enclosure $(H)$ with optically transparent windows made of quartz for incident and scattered beam paths. The measuring temperature was controlled between room temperature and $250^{\circ} \mathrm{C}$ with accuracy of $\pm 1{ }^{\circ} \mathrm{C}$. A He-Ne CW gas laser (A) was used as an incident light source $\left(\lambda_{0}=6328 \AA\right)$. The beam was filtered by an interference filter (B) to eliminate spectral components other than $6328 \AA$. The polarization direction of the laser beam can be varied by rotating a $\lambda / 2$-plate $(\mathrm{D})$. The incident beam was reflected by a mirror $\left(M_{1}\right)$, passed through cone and irradiated on the sample between cone and plate. The scattered light from the sample was passed through the plate (G) and again reflected by a mirror $\left(\mathrm{M}_{2}\right)$, passed through the analyzer $(\mathrm{K})$ and detected by a photographic film (L) or by a TV-camera with a VTR-monitor system described elsewhere $^{16}$ or with a dynamic image-digitizing system recently developed in our laboratory. ${ }^{17}$ The scattering-angular range covered by the present apparatus is typically from 0 to 20 degrees. The incident light intensity level was controlled by rotating a circular neutral density filter $(\mathrm{C}){ }^{18}$

The shear flow was imposed on the sample by applying steady-state rotations or oscillatory rotations to the cone $(\mathrm{E})$, and the torque exerted on the plate $(\mathrm{G})$ was measured by a conventional method. The cone was placed in the incident beam side in order to avoid complexed reflection of the scattered beam at the oblique surface which could occur if the cone was placed in the scattered beam side.

Figure 2 represents the definition of the coordinate system in the flow-SALS experiments, (a) to (d), and polarization conditions
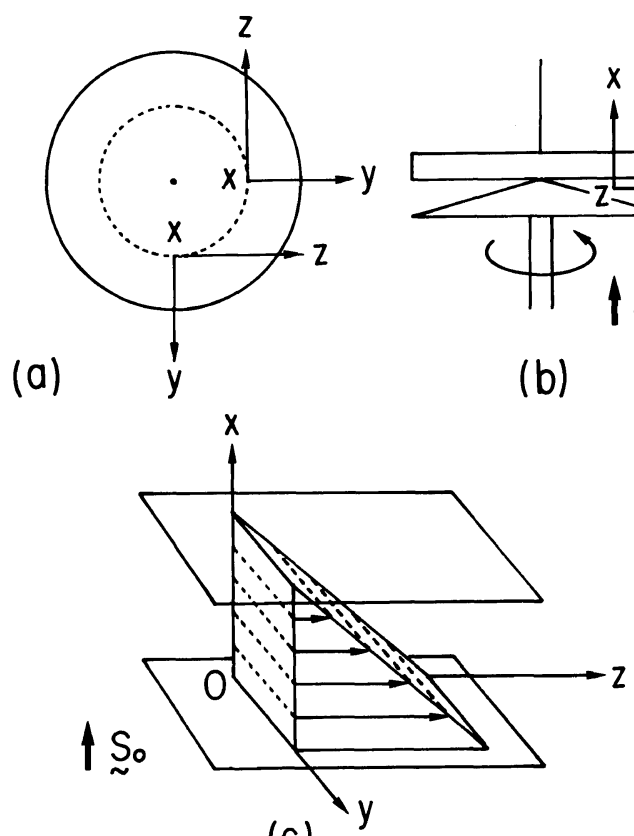

(c)

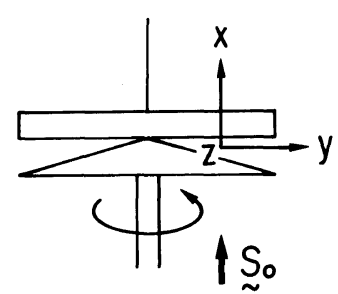

(b)

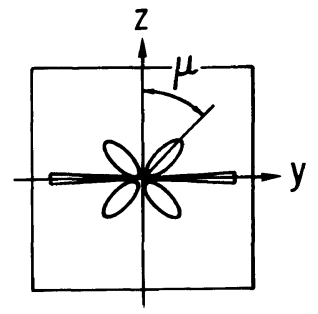

(d)

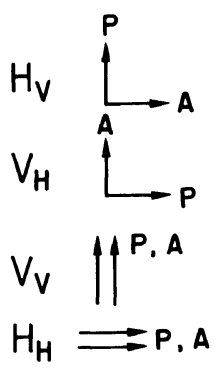

(e)

Figure 2. Definition of the coordinate system in the flow-light scattering experiments (a) to (d) and polarization conditions of the scattering experiment (e). $\mathrm{O} x$ is parallel to the propagation direction of the incident beam (unit vector $\mathrm{S}_{\mathrm{o}}$ ), and $\mathrm{O} z$ is parallel to the flow direction. The velocity gradient of the shear flow exists in the plane parallel to $\mathrm{O} x z$ and the scattering profiles are detected in the plane of $\mathrm{O} y z$ either by photographic films or by a TV camera coupled with a VTR or dynamic image analyzer. 
of the scattering experiment (e). The velocity gradient of the flow exists in the plane parallel to $\mathrm{O} x z$, the incident beam is irradiated through the velocity gradient, parallel to $\mathrm{O} x$, and the small-angle scattering profiles are detected in the plane $\mathrm{O} y z$ perpendicular to the $\mathrm{O} x$ axis. The $\mathrm{O} z$ axis is set parallel to the flow direction and is defined as the vertical direction for the polarized and depolarized light scattering experiments. Upon varying the orientation of the electric vectors of both the $\lambda / 2$-plate and the analyzer, one can detect $H_{\mathrm{V}}, V_{\mathrm{H}}, V_{\mathrm{V}}$, and $H_{\mathrm{H}}$ components of the scattering profiles as indicated in Figure 2(e) where $\mathbf{P}$ and $\mathrm{A}$ denote orientation of the electric vectors of the $\lambda / 2$ plate and the analyzer, respectively.

It should be noted that one has to correct for differences in reflectivities between the vertically and horizontally polarized beams at the mirrors $M_{1}$ and $M_{2}$ for quantitative intensity measurements. ${ }^{34}$

The elastic scattered intensity generally depends on density, concentration, and orientation fluctuations and their spatial correlations as well. ${ }^{19}$ For polymer solutions, liquid crystals, and mixtures, the latter two fluctuations dominate the former. ${ }^{19-23}$ The depolarized components of scattering, $H_{\mathrm{V}}$ and $V_{\mathrm{H}}$, should depend on the orientation fluctuations and correlations, while the polarized components should depend on the concentration fluctuations as well. If the molecular axes tend to orient parallel to the flow direction, then $V_{\mathrm{V}}\left(H_{\mathrm{H}}\right)$ scattering should be more sensitive to orientation (concentration) fluctuations than the concentration (orientation) fluctuations in comparison with $H_{\mathrm{H}}\left(V_{\mathrm{V}}\right)$ scattering. ${ }^{23}$ Thus, both $V_{\mathrm{V}}$ and $H_{\mathrm{H}}$ scattering are recommended to be measured. Both $H_{\mathrm{V}}$ and $V_{\mathrm{H}}$ scatterings are recommended also to be measured in the case when systems exhibit strong optical rotatory power, because the difference depends only on the effect of the optical rotatory power on the scattering. ${ }^{24}$

\section{APPLICATION OF THE FLOW-SALS TO SOLUTION OF RIGID ROD-LIKE MACROMOLECULES}

As a preliminary result to demonstrate prospect of the flow-SALS experiments, we present here rheo-optical investigations of poly $(\gamma-$ methyl D-glutamate) (PMDG, AJICOAT A$\left.200, M_{v}=1.4 \times 10^{5}\right) 20 \mathrm{wt} \%$ in $m$-cresol at room temperature. The concentrations at the A-point and the B-point of the PMDG solution in $m$-cresol were found to be 9 and $17 \mathrm{wt}^{\circ} \%$, respectively. ${ }^{25,26}$

Figure 3 shows the steady-state shear viscosity of the solution as a function of shear rate $\dot{\gamma}$ and corresponding $V_{\mathrm{H}}$ scattering patterns where the flow direction and the polarization directions of the analyzer (A) and the incident beam $(\mathrm{P})$ are indicated in the figure. As shown in the scattering pattern (a), the quiescent solution exhibits a strong smallangle depolarized scattering, the intensity distribution of which is circularly symmetric with respect to the incident beam axis and is a monotonic decay with $\theta$.

Although the solution has a concentration slightly above the B-point it did not exhibit the scattering maximum ${ }^{27,28}$ at $\theta=\theta_{\max }$ which is reciprocally related to the half pitch $S$ of the cholesteric twisting, ${ }^{35}$

$$
2 S \sin \theta_{\max } / 2=\lambda
$$

where $\lambda$ is the wavelength of the light in the medium. The absence of the scattering maximum from the cholesteric pitch may be due to preferential orientation of the cholesteric twisting axes parallel to the $\mathrm{O} x$ axis, even in the quiscent state, or due to the distribution of the pitch. The cholesteric liquid crystals generally form the polydomain structure in which the orientation of $\alpha$-helical molecules is coherent within a given domain but is not coherent between different domains. The domains whose cholesteric twisting axes orient in the plane of $\mathrm{O} y z$ contribute most significantly to the scattering maximum. ${ }^{16,33}$. Therefore the 
Flow Small-Angle Light Scattering

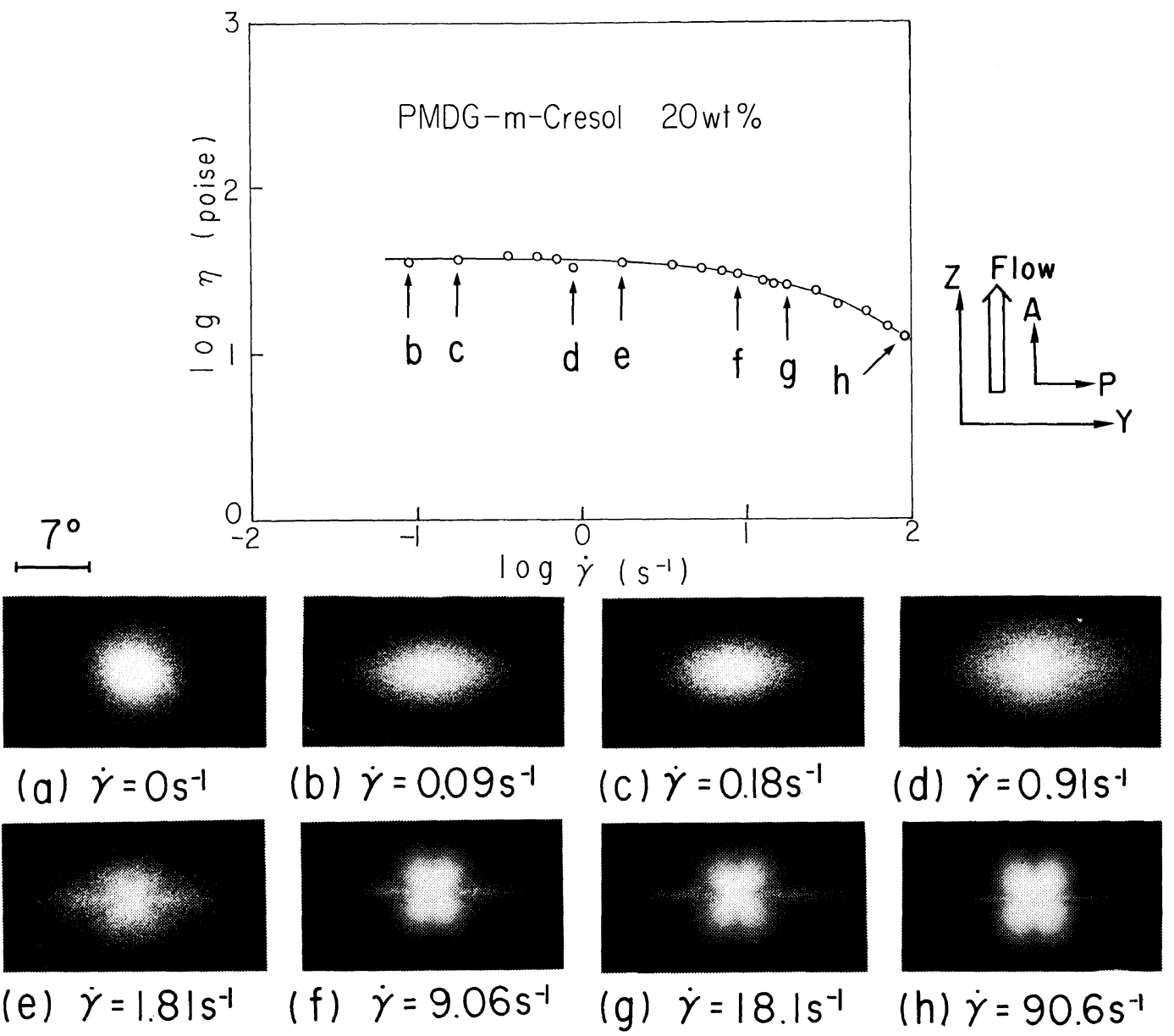

Figure 3. Steady-state shear viscosity $\eta$ and $V_{\mathrm{H}}$ scattering patterns as a function of shear rate $\dot{\gamma}$. The letters (b) to (h) on the curve of $\eta v s . \dot{\gamma}$ correspond to the points where the scattering patterns were taken. The bar on the scattering patterns indicates the length corresponding to the scattering angle $\theta=7^{\circ}$.

absence of the scattering maximum may be a consequence of a preferential orientation of the domains with their twisting axes parallel to the $\mathrm{O} x$ axis. Alternatively, the absence may be a consequence of perturbation in the pitch, simply because the solutions with slightly higher concentrations clearly showed the maximum.

The $V_{\mathrm{H}}$ scattering profiles generally reflect orientation correlation of the $\alpha$-helical molecules. The circularly symmetric scattering profile observed for the quiescent solution (Figure 3(a)) clearly indicates the spherically symmetric orientation correlation. If the twist- ing axes of the domains preferentially orient along the incident beam axis, i.e., $\mathrm{O} x$-axis, then the scattering profiles reflect the orientation correlation of the cholesteric domains in lateral direction, i.e., in the direction perpendicular to the twisting axes.

As the cholesteric liquid crystals of the PMDG $20 \mathrm{wt} \%$ in $m$-cresol are subjected to shear, the depolarized patterns undergo characteristic changes as shown in Figure 3. In the plateau region on the curve of $\eta v s$. $\dot{\gamma}$, corresponding to the region II defined by the works of Asada and his coworkers, ${ }^{29}$ the patterns become elliptical with their long axes 


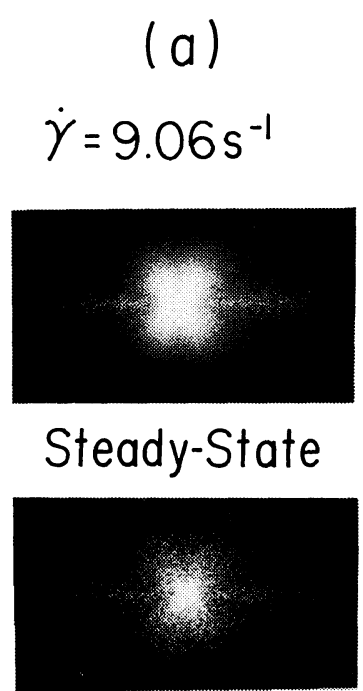

$t=0 \mathrm{~s}$

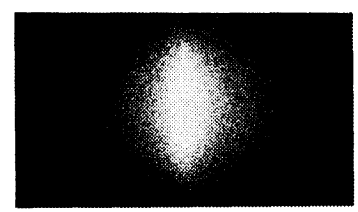

$t=20 s$

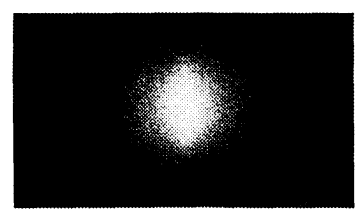

$t=60 \mathrm{~s}$

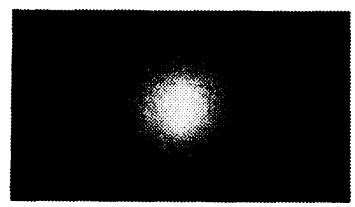

$t=300 s$ (b)
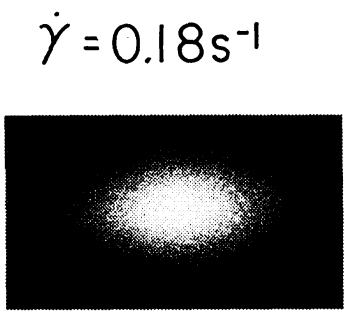

Steady-State

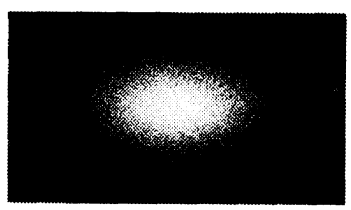

$t=\mathrm{Os}$

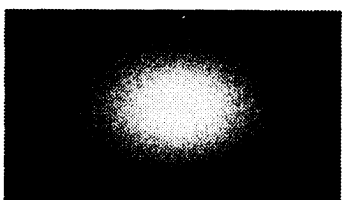

$t=100 \mathrm{sec}$

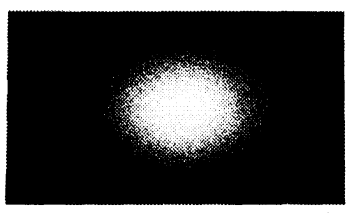

$t=300 s$

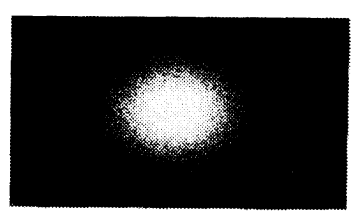

$t=600 \mathrm{~s}$
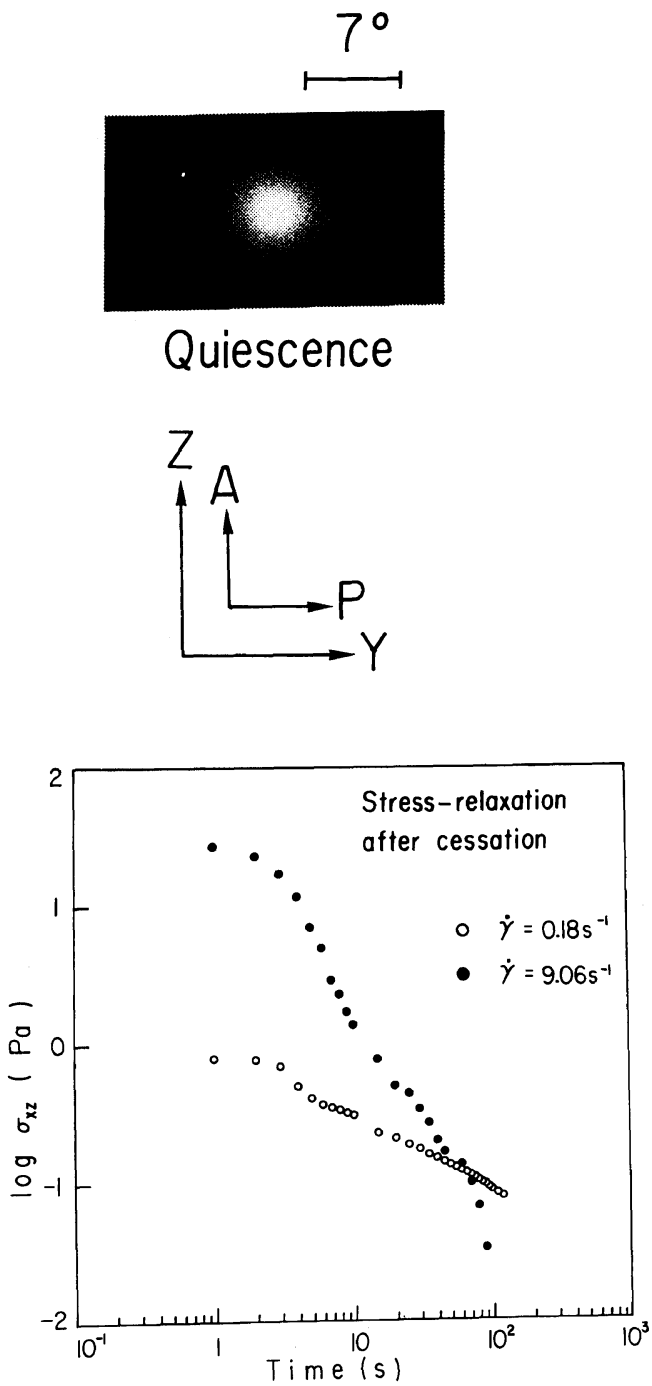

Figure 4. $V_{\mathrm{H}}$ scattering patterns and stress relaxation after the cessations of steady-state shear flows at $\dot{\gamma}=9.06$ (a) and $0.18 \mathrm{~s}^{-1}$ (b). $V_{\mathrm{H}}$ scattering patterns for the solutions in quiescent state and steady-state are also included. The angle mark of $\theta=7^{\circ}$ is indicated on top of the scattering pattern for the quiescent solution.

oriented perpendicular to the flow direction (see the patterns (b) to (d)). On the other hand, in the region of the high shear rate where shear thinning takes place, i.e., in the region corresponding to the region III defined by Asada et al. ${ }^{29}$ the elliptical patterns are transformed 
into the characteristic patterns comprising of (i) a strong and sharp equatorial streak and (ii) $\mathrm{X}$-type scattering patterns quite similar to those observed by Samuels, ${ }^{30}$ Stein et al., ${ }^{31}$ and Hashimoto et al. ${ }^{32}$ for deformed and oriented, optically anisotropic rodlike supermolecular structures. It is our primary objective in this paper to emphasize that there are quite remarkable changes in the supermolecular structures of the rodlike molecules induced by the shear flow. The structural information thus obtained should be useful for the better understanding of the rheological behavior of the materials with ordered but changeable structures.

The supermolecular structures formed under the steady-state shear flow are transformed back to the original structure observed for quiescent solution upon cessation of the steady-state shear flows as typically shown in Figure 4. Thus the change of the supermolecular structures are reversible for the shear rate covered in this work. Figure 4 includes also the $V_{\mathbf{H}}$ pattern for the quiescent solution and stress relaxation $\sigma_{x z}$ with time after the cessation of the steady-state shear flows from $\dot{\gamma}=0.18$ and $9.06 \mathrm{~s}^{-1}$.

The data for $\dot{\gamma}=0.18 \mathrm{~s}^{-1}$ are typical of those for the cessation of the flow in the region II (plateau region), while the data for $\dot{\gamma}=9.06 \mathrm{~s}^{-1}$ are typical of those for the cessation of the flow in the region III (shear thinning region). The data show spectacular features in two major points: (i) the stress relaxation and the recovery of the original supermolecular structure are faster for the solutions sheared with high shear rates than for those sheared with low shear rates, and (ii) mechanism of the structural recovery is distinctly different below and above the critical shear rate $\dot{\gamma}_{\mathrm{c}}$ where the shear thinning starts to become remarkable. The latter feature may be evident from the change of the scattering patterns with time; the recovery from the lower shear rate involves the monotonous and continuous decrease of the axial ratios of the elliptical scattering patterns keeping their long axes in the equatorial direction, while the recovery from the higher shear rates involves rapid disappearance of the equatorial streak, followed by the change of the Xtype pattern into the ellipitical shape with its long axes oriented parallel to the flow direction, the axial ratio of which gradually decreases with time. The preliminary results show the importance of the technique for understanding the physics of flowing polymer systems.

It is beyond the scope of the present paper to give structural interpretations of the depolarized patterns observed under the steady-state shear flows or after the cessation of the shear flows. They will be discussed in a subsequent paper together with investigations of the scattering patterns under different polarization conditions such as $H_{\mathrm{H}}$ and $V_{\mathrm{V}}$ and as a function of the polymer concentration and temperature.

Acknowledgments. The authors are grateful to Iwamoto Seisakusho Co., Ltd., Kyoto, Japan for their technical assistance in constructing the flow-SALS apparatus.

\section{REFERENCES AND NOTES}

1. See, for example, papers in special issue of J. Polym. Sci., C, No. 15 (1966), R. S. Stein and S. Onogi (Ed.), Polym. Sci., ibid., No. 5 (1964).

2. See, for example, papers in special issue of Polym. Eng. Sci., 24 (1984) which is devoted in honor of R. $\mathrm{S}$. Stein upon his receiving the American Chemical Society Award in Polymer Chemistry, Seattle, 1983.

3. J. M. Pochan and D. G. Marsh, J. Chem. Phys., 57, 1193 (1972).

4. D. G. Marsh and J. M. Pochan, J. Chem. Phys., 58, 2835 (1973).

5. D. G. Marsh, J. M. Pochan, and P. Erhardt, J. Chem. Phys., 58, 5795 (1973).

6. T. Asada, Y. Murahashi, and S. Onogi, Nippon Reoroji Gakkaishi, 3, 129 (1975).

7. M. Horio, Ann. Rep., Res. Inst. Chem. Fibers, Jpn., 35, 87 (1978).

8. T. Asada, Y. Murahashi, Y. Kuroki, and S. Onogi, Nippon Reoroji Gakkaishi, 6, 14 (1978).

9. D. G. Baird, A. Ciferri, W. R. Krigbaum, and F. Salaris, J. Polym. Sci., Polym. Phys. Ed., 17, 1694 (1979). 


\section{T. Hashimoto, T. Takebe, and S. Suehiro}

10. H. Aoki, D. Harwood, Y. Lee, J. F. Fellers, and J. L. White, J. Appl. Polym. Sci., 23, 2155 (1979).

11. G. Kiss, T. S. Orrell, and R. S. Porter, Rheol. Acta, 18, 535 (1979).

12. H. Aoki, Y. Onogi, J. L. White and J. F. Fellers, Polym. Eng. Sci., 20, 221 (1980).

13. T. Asada, H. Muramatsu, R. Watanabe, and S. Onogi, Macromolecules, 13, 867 (1980).

14. T. Asada, K. Toda, and S. Onogi, Mol. Cryst. Liq. Cryst., 68, 231 (1981).

15. T. Asada, in "Polymer Liquid Crystals," A. Ciferri, Ed., Chapter 9, Academic Press, N. Y. (1982).

16. T. Hashimoto, S. Ebisu, N. Inaba, and H. Kawai, Polym. J., 13, 701, (1981).

17. S. Suehiro, T. Takebe, T. Hashimoto, and T. Izumitani, Polym. Prepr. Jpn., 33, 2739 (1984).

18. The transmittance continuously varies over 3 to 4 decades by rotation of the circular neutral-density filter.

19. R. S. Stein and P. R. Wilson, J. Appl. Phys., 33, 1914 (1962).

20. R. S. Stein, P. F. Erhardt, S. B. Clough, and G. Adams, J. Appl. Phys., 37, 3980 (1966).

21. T. Hashimoto, and R. S. Stein, J. Polym. Sci., A-2, 8, 1127 (1970).

22. R. S. Stein and T. Hashimoto, J. Polym. Sci., A-2, 8, 1503 (1970).
23. T. Hashimoto, R. E. Prud'homme, and R. S. Stein, $J$. Polym. Sci., Polym. Phys. Ed., 11, 709 (1973).

24. T. Hashimoto, S. Ebisu, and H. Kawai, J. Polym. Sci., Polym. Lett. Ed., 18, 569 (1980).

25. K. Itoh, T. Kajiyama, and M. Takayanagi, Polym. J. 12, 305 (1980).

26. K. Itoh, Ph. D. Thesis, Faculty of Engineering, Kyushu University, Kyushu, Japan, 1980.

27. C. Robinson, Trans. Faraday Soc., 52, 5171 (1955).

28. T. Hashimoto, N. Inaba, S. Ebisu, and H. Kawai, Polym. J., 13, 897 (1981).

29. S. Onogi and T. Asada, Rheology, 1, 127 (1980).

30. R. J. Samuels, J. Polym. Sci., A-2, 7, 1197 (1969).

31. M. B. Rhodes and R. S. Stein, J. Polym. Sci., A-2, 7, 1539 (1969).

32. N. Hayashi, Y. Murakami, M. Moritani, T. Hashimoto, and H. Kawai, Polym. J., 4, 560 (1973).

33. T. Hashimoto, J. Appl. Polym. Sci., Appl. Polym. Symp., 41, 83 (1985).

34. See, for example M. Born and E. Wolf, "Principles of Optics," Pergamon Press, Oxford, 1975, Chapter 1.5.

35. According to the result reported by Itoh, ${ }^{26}$ the $20 \mathrm{wt} \%$ solution should have $\theta_{\max }=2.1^{\circ}$ (in the medium) and $3.3^{\circ}$ (in the air), and hence the scattering maximum should exist in the angular range covered in this experiment. 